\title{
Impact of iron supplementation on schistosomiasis control in Zambian school children in a highly endemic area
}

\author{
Victor Mwanakasale $^{1}$, Seter Siziya ${ }^{2}$, James Mwansa ${ }^{3}$, Artemis Koukounari ${ }^{4}$,
}

\section{Alan Fenwick ${ }^{5}$}

1. Tropical Diseases Research Centre, Ndola, Zambia.

2. University of Zambia, School of Medicine, Lusaka, Zambia.

3. University Teaching Hospital/Schistosomiasis Control Initiative, Lusaka, Zambia.

4. Senior Research Statistician, Schistosomiasis Control Initiative-Imperial College, London, United Kingdom

5. Schistosomiasis Control Initiative-Imperial College, London, United Kingdom

Corresponding author: Dr Victor Mwanakasale, Tropical Diseases Research Centre, P.O.BOX 71769, Ndola, Zambia e-mail addresses: MwanakasaleV@tdrc.org.zm or vicsale@zamtel.zm Mobile phone: 260977-804740, Office phone: 260-212-620737

\section{Abstract \\ Aim: To study impact of once weekly iron supplementation on praziquantel cure rate, Schistosoma haematobium reinfection, and haematological parameters in pupils aged between 9 and 15 years of age in Nchelenge district, Zambia.}

Methods: Pupils in the intervention group received once weekly dose of ferrous sulphate at $200 \mathrm{mg}$ while those in the control received once weekly vitamin $\mathrm{C}$ at $100 \mathrm{mg}$ for up to 9 months. Both study groups received a single dose of praziquantel at baseline.

Results: S haematobium reinfection intensity was significantly lower in boys in the intervention group than in boys in the control group at 6 months $(\mathrm{P}<0.001)$ and 9 months $(\mathrm{P}<0.001)$ of supplementation. Significantly lower $S$ haematobium reinfection intensity was found in girls in the intervention group than in girls in the control group only at 6 months of supplementation $(\mathrm{P}=0.018)$. Boys in the intervention group were $42 \%$ (Adjusted Risk Ratio $=0.58$, $95 \%$ confidence interval $0.39,0.86$ ) less likely to be reinfected with $S$ haematobium than in the control group at 6 months follow up.

Conclusion: Once weekly iron supplementation can decrease $S$ haematobium reinfection after 6 months and should be incorporated into school based schistosomiasis control programs in highly endemic areas.

Clinical trials.gov identifier: NCT 00276224, sponsored by DBL-Institute for Health Research and Development, Denmark.

\section{Introduction}

Schistosomiasis and other parasitic infections contribute significantly to the high prevalence of iron deficiency anaemia in developing countries ${ }^{1-3}$. Other causes of iron deficiency anaemia in tropical countries are dietary deficiency and impaired absorption ${ }^{4}$. 5 . World Health Organisation (WHO) estimates the number of anaemic people worldwide to be a staggering two billion and that approximately $50 \%$ of all anaemia can be attributed to iron deficiency ${ }^{5}$. In areas endemic for schistosomiasis and other tropical diseases such as soil transmitted helminths infection (STH), iron deficiency anaemia accounts for about $50 \%$ of the anaemia in schoolchildren and women ${ }^{2}$. The iron deficiency in schistosomiasis and other tropical diseases such as STH is as a result of blood loss that occurs in these infections.

Chronic iron deficiency anaemia can lead to impaired immunity of individuals infected with schistosomiasis and other tropical diseases ${ }^{6}$. It is widely believed that impaired host immunity as a result of iron deficiency, increases the susceptibility of infected individuals to reinfection with schistosomiasis and other tropical diseases ${ }^{7,8}$.

One method that could be used to correct and prevent the iron deficiency in areas endemic for schistosomiasis and other tropical diseases might be iron supplementation of infected and at risk individuals, respectively ${ }^{1,5}$, . Improved iron levels by iron supplementation in infected and at risk individuals might improve their immunity leading to increased resistance to reinfection with schistosomiasis and STH infections.

In this article we present findings from a school-based trial of placebo controlled once weekly iron supplementation entitled "Iron supplementation in schistosomiasis and soil transmitted helminths control programs in Zambia". This trial compared once weekly iron supplementation with a placebo in terms of the following endpoints; praziquantel cure rate of $S$ haematobium determined at 3 months post treatment, $S$ haematobium reinfection intensities and rates at 6 and 9 months post treatment, changes in mean haemoglobin levels and anaemia prevalence between baseline and after 9 months of iron supplementation, and mean number of doses of iron/placebo received by each follow up times at 6 and 9 months.

\section{Materials and methods}

\section{Study design, area and population}

The study was a school-based placebo-controlled intervention trial conducted in Nchelenge district in Luapula province of Zambia. It was conducted between September 2005 and July 2006. The two schools, Kenani Upper Basic school and Chandwe Middle Basic School, were selected as the study schools for this trial. These two schools lie 3 kilometres apart, off the shores of Lake Mweru. Kenani school was the intervention school where school children aged 9-15 years, of either sex, in Grades 2,3, and 4, and present on the day of screening formed the intervention group. These children received once weekly iron supplementation for a maximum nine months, interrupted by two one-month school holidays. Chandwe was the control school where children in Grades 2 and 3, aged 9-15 years, of either sex, and present on the day of screening formed the control group that received a placebo (vitamin C) once weekly for a maximum nine months. This age group of 9-15 years was selected because it is ideal for the monitoring and evaluation of school based schistosomiasis and STH infections control programs ${ }^{10}$.

Vitamin C was only given to the control group and not to the intervention group so as to make the two groups comparable 
in terms of number of tablets swallowed by the study pupils during the study period.

The only source of $S$ haematobium infected waters for both schools and the surrounding community is Lake Mweru. These two study schools were selected because the community in which they are situated is homogenous in terms of the cultural, tribal and socio-economic status of households. The economic activities in this community are fishing, trading, and subsistence farming.

\section{Sample size}

The sample size was calculated using the Statcalc programme in Epi Info version 6.04c (Centres for Disease Control and Prevention, Atlanta, GA). A sample size of 240 children in each of the study schools was calculated based on the study objectives of comparing the effect of weekly iron supplementation in children in both study schools using the following parameters (Confidence Interval of 95\%, Power of $80 \%$, Unexposed: Exposed ratio of 1:1, Disease in the exposed of $20 \%$, Risk ratio of 2.0 , and Odds ratio of 2.25).

\section{Baseline survey}

Written informed consent was obtained from parents/ guardians before enrolment of the study pupils. Pupils had also to give a verbal consent before being enrolled into the study and those who gave consent were then requested to submit one urine sample as well as blood drawn from their finger for haemoglobin analysis by the study nurse using a hemoglobinometer called Hemocue $\mathrm{Hb} 201+$ (HemoCue AB, Angelholm, Sweden).

The study pupils were then asked to submit a second urine sample the following day. The urine samples were examined for $S$ haematobium ova by the study laboratory technicians using the urine filtration microscopy method (Vestergaard Frandsen A/S, Denmark).

\section{Treatment and iron supplementation}

All the pupils participating in this study were given a single dose of praziquantel regardless of their infection status after submitting both urine samples and urine examination completed. The dosage for the praziquantel was calculated using the dose pole ${ }^{11}$. Once weekly iron supplementation in the intervention group and once weekly placebo (vitamin C) to all study pupils was provided on the day after administration of praziquantel. The class teachers in the selected grades administered the once weekly iron/vitamin C supplementation for nine months under direct observation. The iron supplementation was given in form of an oral tablet of ferrous sulphate B.P of $200 \mathrm{mg}$ ( $65 \mathrm{mg}$ of ferrous iron) once per week while vitamin $C$ was given in form of an oral tablet of Ascorbic Acid B.P of $100 \mathrm{mg}$ once per week, respectively.

\section{Parasitological and haematological follow up}

All the study pupils were followed up at three, six, and nine months post praziquantel treatment where they were requested to submit again two urine samples collected on two consecutive days. Methodology used here was exactly identical to that employed at baseline. The six and nine months sample points were used to assess effect of duration of iron supplementation on $S$ haematobium reinfection.

No additional praziquantel treatment was provided at either 3 or 6 months follow-up.

End of study treatment. All the study pupils were given a second dose of praziquantel at the 9 months follow up regardless of their $S$ haematobium infection status.

\section{Statistics}

Data were double entered in Epi Info v 6.04c. Data analysis was done in Epi Info, Stata version 6.0 (Stata Corp, College Station, TX), and SPSS. The statistical tests used in analysis were chi-square test for comparison of proportions and t-test for comparison of means. Primary outcome measures used to assess the impact of once weekly iron supplementation were parasitological cure rate of praziquantel for $S$ haematobium infection at 3 months post treatment, $S$ haematobium reinfection rate and intensity at 6 months, $S$ haematobium reinfection rate and intensity at 9 months, and mean haemoglobin levels and anaemia prevalence at 9 months. Parasitological cure rate of praziquantel for $S$ haematobium infection was defined as the proportion of individuals who were diagnosed as negative from microscopic examination of their urine. The measure used for the egg count for $S$ haematobium infection for each individual was the maximum number of eggs counted in any of the two urine samples submitted at each of the screening times and was recorded as egg/10 mL. S haematobium reinfection at 6 months was defined as the presence of $S$ haematobium ova in urine at 6 months in children who had been diagnosed as negative at three months and having had ova at baseline. $S$ haematobium reinfection at 9 months was defined as the presence of $S$ haematobium ova in urine at 9 months in children who had been diagnosed as negative at 3 months (regardless of the $S$ haematobium infection status at 6 months) and had ova at baseline. Anaemia was defined according to WHO guidelines as haemoglobin levels less than $11 \mathrm{~g} / \mathrm{dL}^{12}$.

Geometric mean egg count was used to summarise the intensity of $S$ haematobium in intervention and control groups and it was calculated among all the children-positive and negative. Stratified analysis was conducted considering age as a possible confounder for each sex. An unadjusted estimates [Odds Ratio (OR), and Relative Risk (RR)] as well as adjusted estimates [Adjusted Odds Ratio (AOR), and Adjusted Relative Risk (ARR)] are presented together with their 95\% Confidence Interval (CI).

\section{Results}

218 pupils in the intervention group and 228 pupils in the control group had complete available data for statistical analysis.

The intervention and control groups were comparable in terms of the sex distribution of the study pupils. The total number of boys was $104(47.7 \%)$ out of 218 pupils in the intervention group, and $111(48.7 \%)$ out of 228 in the control group $(p=0.836)$. However, pupils in the control group tended to be younger than those in the intervention group $(\mathrm{p}<0.001)$, mean age in the control group being 10.5 years $(95 \%$ CI $10.2,10.7)$ while that of the intervention group was 11.1 years $(95 \%$ CI 10.8, 11.3). Overall $48(22.0 \%)$ 
Table 1: Comparison of baseline, cure rate (3 months) and re-infection rates for S.haematobium at 6 and 9 months follow up between the intervention and control groups.

\begin{tabular}{|c|c|c|c|c|c|c|c|c|}
\hline & \multicolumn{4}{|c|}{ Male } & \multicolumn{4}{|c|}{ Female } \\
\hline \multirow[t]{2}{*}{ Follow-up } & & $n(\%)$ & Crude risk estimate & $\begin{array}{l}\text { Adjusted risk } \\
\text { estimate* }\end{array}$ & Tota & $\mathrm{n}(\%)$ & Crude risk estimate & $\begin{array}{l}\text { Adjusted risk } \\
\text { estimate* }^{*}\end{array}$ \\
\hline & & & OR $(95 \% \mathrm{Cl})$ & AOR $(95 \% \mathrm{Cl})$ & & & OR $(95 \% \mathrm{Cl})$ & AOR $(95 \% \mathrm{Cl})$ \\
\hline \multicolumn{9}{|l|}{ Baseline } \\
\hline Intervention & & $93(89.4)$ & $0.40(0.11,1.32)$ & $0.43(0.14,1.29)$ & 114 & $103(90.4)$ & $0.60(0.20,1.76)$ & $0.49(0.18,1.34)$ \\
\hline \multirow[t]{2}{*}{ Control } & & $106(95.5)$ & 1 & 1 & 117 & $110(94.0)$ & 1 & 1 \\
\hline & & & RR $(95 \% \mathrm{Cl})$ & ARR $(95 \% \mathrm{Cl})$ & & & $\mathrm{RR}(95 \% \mathrm{Cl})$ & ARR $(95 \% \mathrm{Cl})$ \\
\hline \multicolumn{9}{|l|}{3 months } \\
\hline Intervention & 58 & $2(3.4)$ & $1.60(0.23,11.07)$ & $1.44(0.26,7.88)$ & 54 & $3(5.6)$ & $2.56(0.44,14.82)$ & $4.13(0.46,36.67)$ \\
\hline Control & & $2(2.2)$ & 1 & 1 & 92 & $2(2.2)$ & 1 & 1 \\
\hline \multicolumn{9}{|l|}{6 months } \\
\hline Intervention & 46 & $19(41.3)$ & $0.59(0.41,0.87)$ & $0.58(0.39,0.86)$ & 44 & $29(65.9)$ & $1.01(0.77,1.32)$ & $0.79(0.74,1.26)$ \\
\hline Control & & $50(69.4)$ & 1 & 1 & 72 & $47(65.3)$ & 1 & 1 \\
\hline \multicolumn{9}{|l|}{9 months } \\
\hline Intervention & 31 & $17(54.8)$ & $0.71(0.51,1.00)$ & $0.73(0.52,1.03)$ & 27 & $16(59.3)$ & $0.83(0.58,1.18)$ & $0.79(0.56,1.12)$ \\
\hline Control & 74 & $57(77.0)$ & 1 & 1 & 63 & 45 (71.4) & 1 & 1 \\
\hline
\end{tabular}

* adjusted for age

of pupils in the intervention group compared to $99(43.4 \%)$ in the control group were below the age of 10 years.

Infection rates of $S$ haematobium at baseline, 3 months, and at 9 months follow up were similar between the intervention and control groups among boys. However, at 6 months follow-up, boys in the intervention group were $42 \%$ (ARR $=0.58,95 \%$ CI $0.39,0.86)$ less likely to have had $S$ haematobium reinfection compared to boys in the control group. Among girls, infection rates of $S$ baematobium were similar between the two groups at baseline and at all follow up surveys. These results are shown in Table 1. When comparison was made between the study groups, without adjusting for age and sex, the $S$ haematobium reinfection rates were significantly higher in the control group compared to the intervention group at 6 months $(\mathrm{P}=0.032)$ and at 9 months $(\mathrm{P}=0.015)$ as demonstrated in Figure 1.

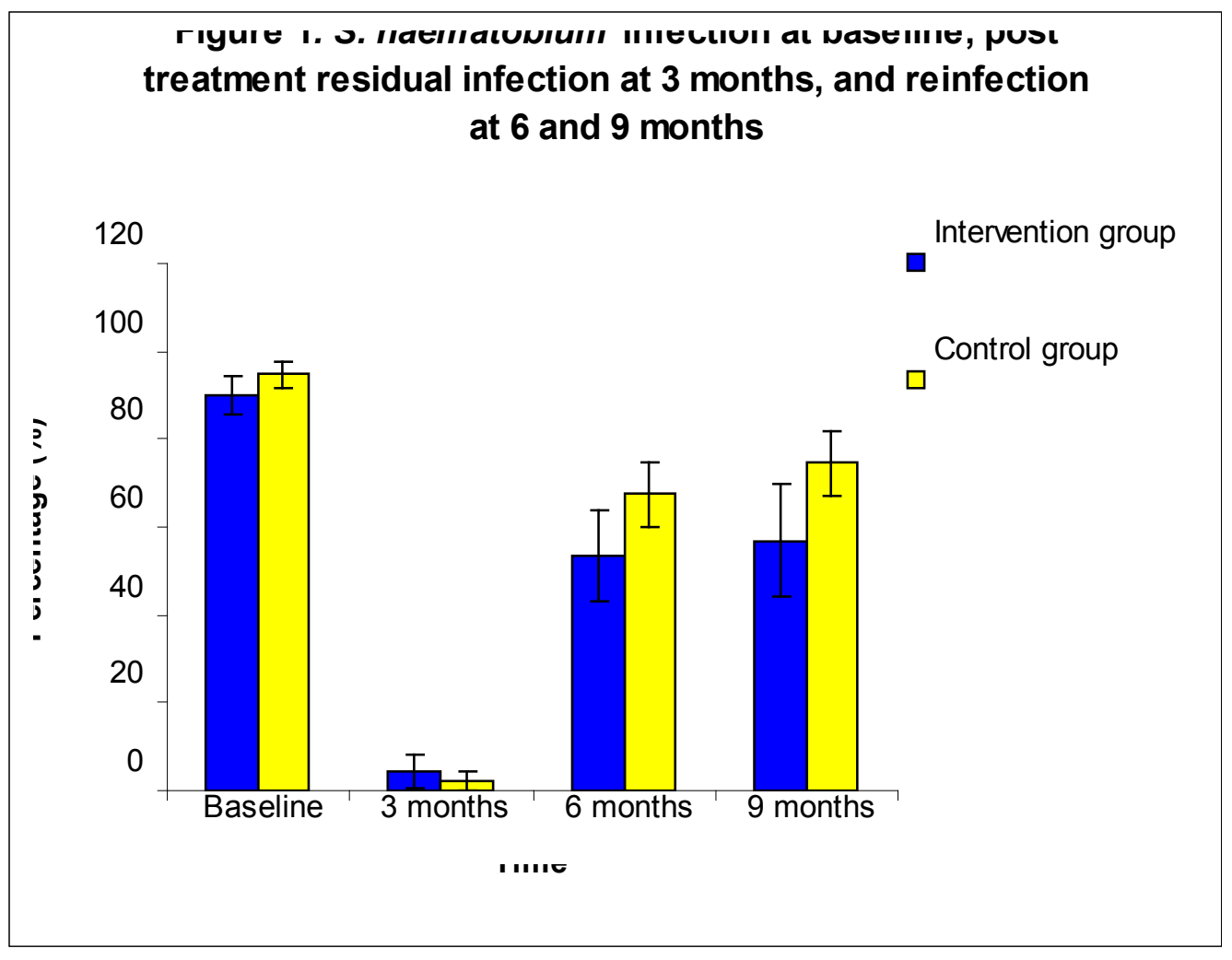


Table 2: Comparison of S. haematobium infection intensity between

the intervention and control group at each survey.

\begin{tabular}{|c|c|c|c|c|c|c|}
\hline \multirow[b]{2}{*}{ Follow-up } & \multicolumn{3}{|c|}{ Male } & \multicolumn{3}{|c|}{ Female } \\
\hline & Total & Mean (SD) & $\begin{array}{l}p \\
\text { value }\end{array}$ & Total & \begin{tabular}{|l|} 
Mean \\
(SD) \\
\end{tabular} & $\begin{array}{l}p \\
\text { value }\end{array}$ \\
\hline \multicolumn{7}{|l|}{ Baseline } \\
\hline Intervention & 104 & $1.699(0.791)$ & 0.164 & 114 & $\begin{array}{l}1.647 \\
(0.752)\end{array}$ & 0.280 \\
\hline Control & 111 & $1.842(0.714)$ & & 117 & $\begin{array}{l}1.754 \\
(0.744)\end{array}$ & \\
\hline \multicolumn{7}{|l|}{3 months } \\
\hline Intervention & 58 & $0.050(0.293)$ & 0.612 & 54 & \begin{tabular}{|l|}
0.052 \\
$(0.216)$
\end{tabular} & 0.151 \\
\hline Control & 93 & $0.029(0.213)$ & & 92 & \begin{tabular}{|l|}
0.014 \\
$(0.098)$
\end{tabular} & \\
\hline \multicolumn{7}{|l|}{\begin{tabular}{|l|}
6 months \\
\end{tabular}} \\
\hline Intervention & 46 & $0.407(0.584)$ & $<0.001$ & 44 & \begin{tabular}{|l|l}
0.652 \\
$(0.633)$ \\
\end{tabular} & 0.018 \\
\hline Control & 72 & $1.028(0.875)$ & & 72 & \begin{tabular}{|l|}
1.025 \\
$(0.907)$ \\
\end{tabular} & \\
\hline \multicolumn{7}{|l|}{9 months } \\
\hline Intervention & 31 & $0.552(0.581)$ & $<0.001$ & 27 & \begin{tabular}{|l|}
0.826 \\
$(0.793)$
\end{tabular} & 0.119 \\
\hline
\end{tabular}

Table 2 shows the distribution of $S$ baematobium intensity between the study groups for each sex at each survey. Intensities of $S$ baematobium infection were comparable between the intervention and control group at baseline and at subsequent follow up surveys apart from the 6 months follow up among girls. At the 6 months follow up, the intensity of $S$ haematobium reinfection was heavier in the control group than in the intervention group among girls. Among boys, no significant differences in $S$ baematobium infection intensities were observed between the intervention and control group at baseline and at the 3 months follow up. At subsequent follow up surveys $(6$ and 9 months follow up), intensities of infection were heavier among boys in the control group compared to the intervention group. Unadjusted comparison between the two study groups, showed that the intensities of $S$ baematobium reinfection were significantly heavier in the control group compared to the intervention group at 6 months $(\mathrm{P}<0.01)$ and at 9 months $(\mathrm{P}<0.01)$, as shown in Figure 2.

The prevalence rates of anaemia were comparable between the intervention and control group among boys at both the baseline, and at 9 months follow up. At 9 months follow up, among girls, the control group had a significantly higher prevalence of anaemia than the intervention group; adjusted bivariate analysis for age showed that this association was no longer significant (Table 3). At 9 months follow up, unadjusted comparison between the two study groups, the prevalence of anaemia was higher in the control group compared to the intervention group $(\mathrm{P}=0.014)$.

Mean haemoglobin ( $\mathrm{Hb}$ ) levels (Table 4) were not significantly different between the intervention and control group at both baseline, and at 9 months follow up when adjusted analysis was performed. However, when unadjusted analyses were performed the mean haemoglobin levels were significantly higher in the intervention group compared to the control group at 9 months follow up $(\mathrm{P}=0.014)$, as shown in Figure 3.

Unadjusted analyses also showed that mean number of doses of iron/placebo supplementation received by 6 and 9 months were not significantly different between the two groups $(\mathrm{P}=0.278$ and $\mathrm{P}=0.524$, respectively), as shown in

\section{Figure 2. S. haematobium intensity (geometric mean) of infection at baseline and 3 months and reinfection at 6 and 9 months follow ups}

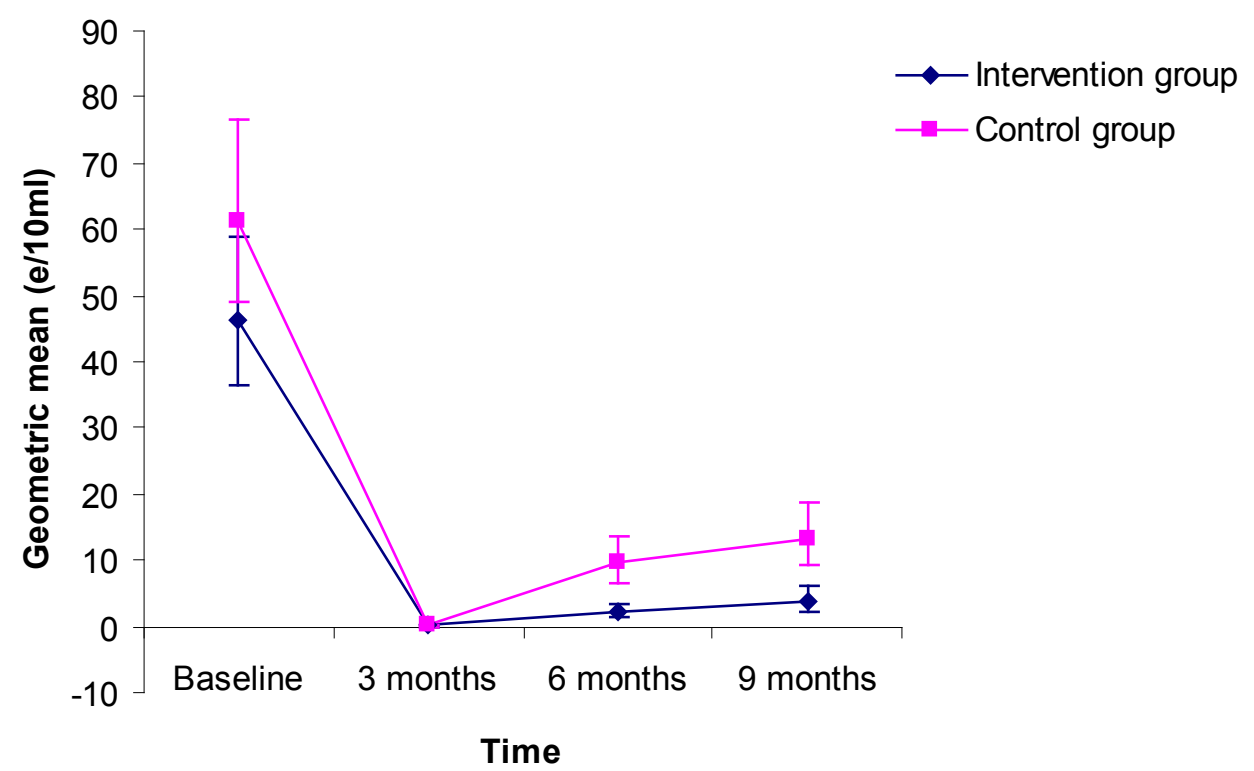


Table 3: Comparison of prevalence of anaemia between intervention and control group

\begin{tabular}{|c|c|c|c|c|c|c|c|c|}
\hline & \multicolumn{4}{|c|}{ Male } & \multicolumn{4}{|c|}{ Female } \\
\hline Follow-up & Total & $\mathrm{n}(\%)$ & Crude risk estimate & $\begin{array}{l}\text { Adjusted risk } \\
\text { estimate }^{*}\end{array}$ & Total & $\mathrm{n}(\%)$ & Crude risk estimate & $\begin{array}{l}\text { Adjusted risk } \\
\text { estimate }^{*}\end{array}$ \\
\hline & & & OR $(95 \% \mathrm{Cl})$ & AOR $(95 \% \mathrm{Cl})$ & & & OR $(95 \% \mathrm{Cl})$ & AOR $(95 \% \mathrm{Cl})$ \\
\hline \multicolumn{9}{|l|}{ Baseline } \\
\hline Intervention & 80 & $17(21.3)$ & $1.03(0.46,2.34)$ & $1.04(0.58,1.89)$ & 73 & $11(15.1)$ & $0.42(0.17,0.99)$ & $0.50(0.23,1.13)$ \\
\hline \multirow[t]{2}{*}{ Control } & 87 & $18(20.7)$ & 1 & 1 & 84 & $25(29.8)$ & 1 & 1 \\
\hline & & & RR $(95 \% \mathrm{Cl})$ & ARR $(95 \% \mathrm{Cl})$ & & & RR $(95 \% \mathrm{Cl})$ & $\operatorname{ARR}(95 \% \mathrm{Cl})$ \\
\hline \multicolumn{9}{|l|}{9 months } \\
\hline Intervention & 80 & $3(3.8)$ & $0.36(0.10,1.29)$ & $0.34(0.08,1.35)$ & 73 & $3(4.1)$ & $0.31(0.09,1.08)$ & $0.31(0.09,1.10)$ \\
\hline Control & 87 & $9(10.3)$ & 1 & 1 & 84 & $11(13.1)$ & 1 & 1 \\
\hline
\end{tabular}

* adjusted for age

Table 4: Comparison of haemoglobin levels between the intervention and control group

\begin{tabular}{|l|l|l|l|l|l|l|}
\hline & \multicolumn{3}{|c|}{ Male } & \multicolumn{3}{c|}{ Female } \\
\hline Follow-up & Total & Mean (SD) & $p$ value & Total & Mean (SD) & $p$ value \\
\hline Baseline & & & & & & \\
\hline Intervention & 80 & $11.79(1.136)$ & 0.793 & 73 & $11.86(1.085)$ & 0.215 \\
\hline Control & 87 & $11.83(1.280)$ & & 84 & $11.63(1.241)$ & \\
\hline & & & & & & \\
\hline 9 months & & & & & & \\
\hline Intervention & 80 & $12.98(1.115)$ & 0.104 & 73 & $13.02(1.116)$ & 0.067 \\
\hline Control & 87 & $12.66(1.331)$ & & 84 & $12.65(1.354)$ & \\
\hline & \multicolumn{7}{|l|}{} & & & & \\
\hline Change in Hb from baseline to 9 months follow up & & & \\
\hline \multicolumn{7}{|l|}{} \\
\hline
\end{tabular}

Figure 4.

\section{Discussion}

There is still controversy over the benefits of iron supplementation in the control of infections in endemic areas. Whereas certain studies have demonstrated positive impact of iron supplementation on the control of infections such as schistosomiasis ${ }^{13}$ and upper respiratory tract infections as well as diarrhoeal diseases ${ }^{14,15}$, other studies have found no benefits of iron supplementation on the control of schistosomiasis and other helminths ${ }^{16}$ as well as malaria infections ${ }^{17,18}$.

The two study schools are located in an area highly endemic for $S$ haematobium infection as evidenced by the high prevalence at baseline (the prevalence of $S$ haematobium infection in the intervention group was $89.9 \%$ while that in the control group was $94.8 \%$ ). In our study, iron supplementation was administered by the school teachers. The mean number of doses of iron supplementation received in the intervention group was comparable with that of placebo in the control group at both 6 and 9 months follow up times. This observation might imply that there were no significant factors such as side effects to the intervention drug, iron, that could have made class attendance in the intervention group significantly lower than in the control group through absenteeism. The high mean number of doses of the intervention and the placebo received by the study pupils at both follow up times indicated that school based iron supplementation programs could be feasible in areas highly endemic for $S$ haematobium infection.

Once weekly iron supplementation had no impact on praziquantel cure rate for $S$ haematobium infection determined at 3 months post treatment. This observation, it could be argued, was as expected as the period of iron supplementation was too short for any effect to be seen on the immune system $^{19}$. Furthermore, the high praziquantel cure rates for $S$ haematobium observed in both study groups (96.6\% for the intervention group and $97.8 \%$ for the control group) were acceptable considering the high prevalence of $S$ haematobium in the study area.

The demonstration of significant reduction in the intensity and rate of $S$ haematobium reinfection after 6 months of iron supplementation in the intervention group when compared 

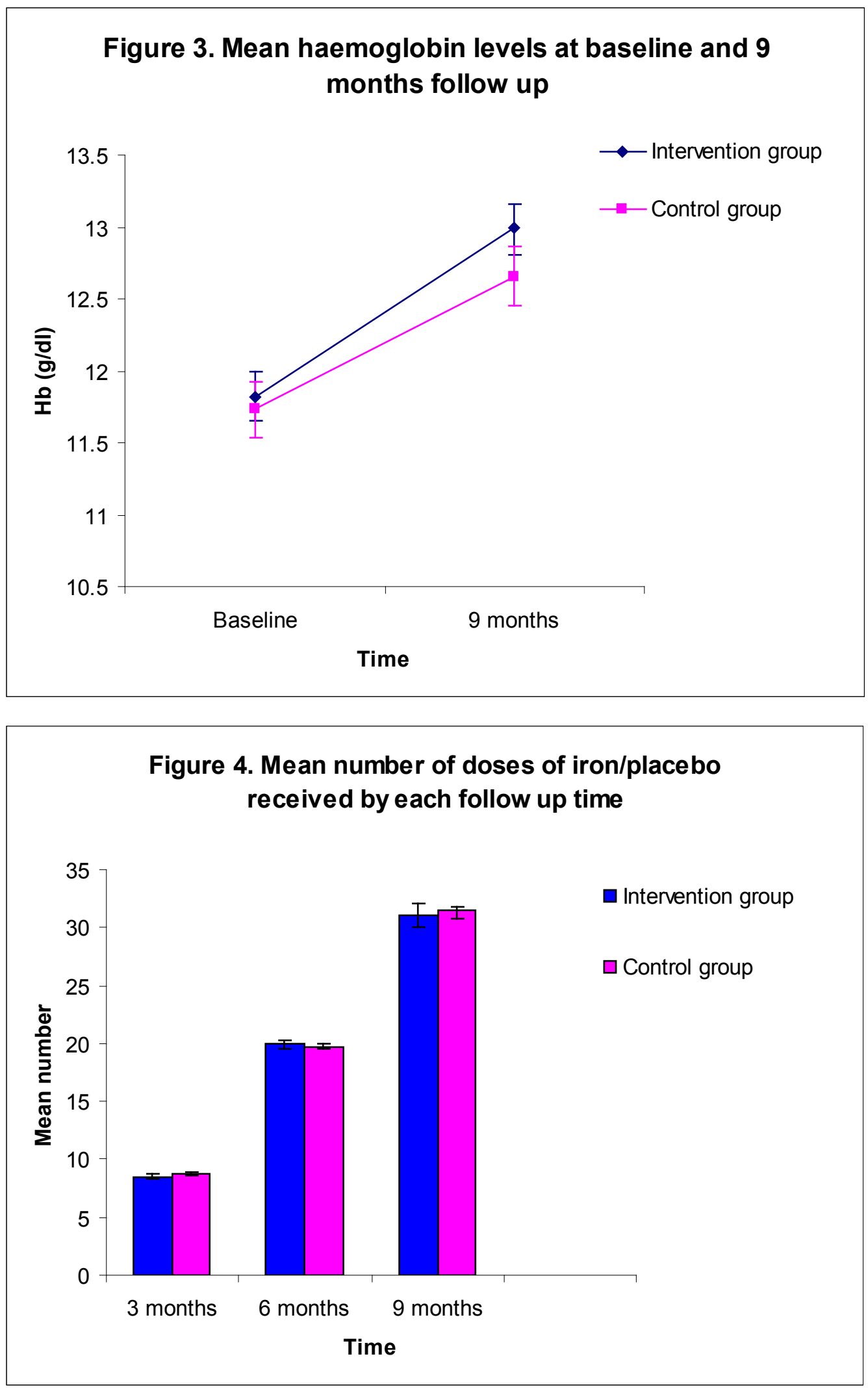

with the placebo group in our study implies that there was a positive impact of iron on the host immune system towards new infection. It may be argued that increased iron supplementation might have enhanced the immune system in destroying new invading schistosomes and thereby reducing reinfection rate and intensity of $S$ haematobium ${ }^{20,13}$. We do not think there were any confounding factors, for example nutritional status differences between the two study groups, that could explain this observation as the two study groups were comparable in terms of geographical location of the two schools, various diseases prevalence in the community, health care provision in the community, and the social and economic status of the study pupils. The pupils in the two study groups belonged to one and same community.

Our study failed to demonstrate significant increase in mean haemoglobin levels and reduction in anaemia prevalence after 9 months of iron supplementation in the intervention group when compared with the control group. The probable explanation for this observation is that the period of iron supplementation might have been too short for any significant effect on haemoglobin levels and therefore anaemia. Further 
research is therefore warranted to establish the impact of longer duration of once weekly iron supplementation on haemoglobin levels and anaemia.

Even though iron supplementation was insufficient in significantly increasing haemoglobin levels and thereby not significantly reducing anaemia, it was sufficient in correcting the iron deficiency and thereby having a positive impact on $S$ baematobium re-infection. The explanation for this observation is that the levels of iron required to have a positive impact on $S$ haematobium re-infection is lower than the levels required to significantly increase the haemoglobin levels and therefore significantly reduce the anaemia. The causation of anaemia in our study children most likely was multifactorial, with iron deficiency being one of them. Iron supplementation corrected the iron deficiency which was enough to increase resistance to $S$ haematobium re-infection but not enough to correct the iron deficiency anaemia. No studies have been conducted before in our study children to establish the iron stores of these children.

In conclusion, this study has demonstrated that in an area highly endemic for $S$ haematobium infection, school based once weekly iron supplementation in the control of schistosomiasis is feasible and can reduce $S$ haematobium reinfection significantly after 6 months of supplementation. We recommend that once weekly iron supplementation should be incorporated into school based schistosomiasis control programs in highly endemic areas.

\section{Funding}

Funding for the study whose data has been presented in this article was by Bill and Melinda Gates Foundation through the Schistosomiasis Control Initiative (SCI) at Imperial College, London, United Kingdom.

\section{Acknowledgements}

We are grateful to Bill and Melinda Gates Foundation for having funded the study from whose data we have presented in this article. We wish to thank Professor Niels Ørnbjerg, Director of DBL-Institute for health research and development, Denmark, for having facilitated the release of the research funds for the study. We also thank Dr E Kafwembe, Director of Tropical Diseases Research Centre, Ndola, Zambia, for the support he rendered to the study. We are also grateful to Dr Kitengi, Medical officer at St Paul's Mission Hospital, Nchelenge, for having allowed us to use the hospital's laboratory for the analysis of our study samples. We are also indebted to all the teachers at the two study schools for all their effort in ensuring the success of the study. Finally we wish to thank the two laboratory technologists that examined the urine samples in the field and the study nurse who analysed the blood samples.

\section{References}

1. Partnership for Child Development, 2004. Iron deficiency anaemia. Available from URL: http:/ /www.schoolsandhealth.org

2. Asobayire F S, Adou P, Davidsson L, Cook JD, Hurrell RF, 2001. Prevalence of iron deficiency with and without concurrent anaemia in population groups with high prevalence of malaria and other infections: a study in Cote d'Ivoire. American Journal of Clinical Nutrition 74: 776-782.

3. Van den Broek NR, Letsky EA, 2000. Aetiology of anaemia in pregnancy in South Malawi. American Journal of Clinical Nutrition 72: $247 \mathrm{~S}-256 \mathrm{~S}$

4. Centers for Disease Control and Prevention, 1998. Recommendations to prevent and control iron deficiency in the United States. Morbidity and mortality Weekly Report 47.

5. World Health Organisation, 2004. Focusing on anaemia: towards an integrated approach for effective anaemia control. Joint statement by WHO and UNICEF

6. Chandra RK, 1975. Impaired Immunocompetence Associated with Iron Deficiency. Journal of Paediatrics 86: 899-902.

7. Failla ML, 2003. Trace elements and host defense: recent advances and continuing challenges. Journal of Nutrition May 1335 Suppl 1: 1443S1447S.

8. Hughes S, Kelly P, 2006. Interactions of malnutrition and immune impairment, reference to immunity against parasites. Parasite Immunology November 28 11: 577-588.

9. Olsen A, Nawiri J, Friis H, 2000. The impact of iron supplementation on re-infection with intestinal helminths and Schistosoma mansoni in western Kenya. Transactions of the Royal Society of Tropical Medicine and Hygiene 94: 493-499.

10. Montressor A, Crompton DWT, Bundy DAP, Hall A, Savioli L, 1998. Guidelines for the evaluation of soil-transmitted helminthiasis and schistosomiasis at community level. WHO/CTD/SIP/98.1.

11. World Health Organisation, 2004. Action Against Worms-Praziquantel dose pole. Available from URL: http://www.who.int/wormcontrol

12. United Nations Children's Funds, United Nations University, World Health Organisation, 2001. Iron Deficiency Anaemia Assessment, Prevention and Control. A Guide for Programme Managers. World Health Organisation, Geneva.

13. Taylor M, Jinabhai CC, Couper I, Kleinschmidt I, Jogessar VB, 2001. The effect of different anthelmintic treatment regimens combined with iron supplementation on the nutritional status of schoolchildren in KwaZulu-Natal, South Africa: a randomised controlled trial. Transactions of the Royal Society of Tropical Medicine and Hygiene. Mar-Apr 95 2: 211-6.

14. Berger J, Dyck JL, Galan P, Aplogan A, Schneider D, Traissac P, Hercberg S, 2000. Effect of daily iron supplementation on iron status, cell-mediated immunity, and incidence of infections in 6-36 month old Togolese children. European Journal of Clinical Nutrition.Jan; 54(1): 29-35.

15. de Silva A, Atukorala S, Weerasinghe I, Ahluwalia N, 2003. Iron supplementation improves iron status and reduces morbidity in children with or without upper respiratory tract infections: a randomised controlled study in Colombo, Sri Lanka. American Journal of Clinical Nutrition. Jan;77(1): 234-41.

16. Stephenson LS, Latham MC, Kurz KM, Miller D, Kinoti SN, Oduori ML, 1985. Urinary iron loss and physical fitness of Kenyan children with urinary schistosomiasis. American Journal of Tropical Medicine and Hygiene. Mar 34 2: 322-30.

17. Murray MJ, Murray AB, Murray CJ, 1978. The adverse effect of iron repletion on the course of certain infections. British Medical Journal. 2:1113-1115.

18. Oppenheimer SJ, 2001. Iron and its relation to immunity and infectious disease. Journal of Nutrition. 131:616s-63s

19. Solon SF, Fajutrao L, Solon AJ, Sarol N J, Wambangco T, Femin S L. Weekly iron supplementation for 1 to 5 year old children, an economical and effective intervention for anaemia prevention. Philippine Association of Nutrition, Inc. Available from URL:http:// www.fnri.dost.gov.ph/pan/recommends.htm

20. Berger LL. Trace minerals: Keys to immunity. Available from URL: http://www.saltinstitute.org/STM-1.html 